\title{
EL TRABAJADOR SOCIAL EN LA DIRECCIÓN DE UN CENTRO DE SERVICIOS SOCIALES
}

\section{VÍCTOR M. GIMÉNEZ BERTOMEU}

Trabajador Social. Coordinador del Centro Social n. ${ }^{\circ}$ 4. Servicios Sociales Municipales del Ayuntamiento de Alicante.

\section{RESUMEN}

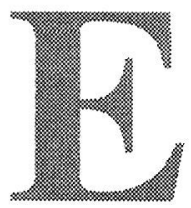

1 presente trabajo pretende acercarse a la figura del trabajador social como director de organizaciones de Servicios Sociales, y más específicamente de Centros Sociales o de Servicios Sociales.

En primer lugar, se encuadra el contexto desde el que se hace la reflexión, delimitando los conceptos de partida y caracterizando las organizaciones donde vamos a situar al trabajador socialdirector.

En segundo lugar, se aborda el contenido de la función directiva en los Centros de Servicios Sociales. Partiendo de la contraposición entre un modelo innovador y una gerencia tradicional, por un lado, se identifican las funciones del director, con especial incidencia en la utilidad de la supervisión en este ámbito, y por otro, las características necesarias para una dirección eficaz.

Por último, se recogen aquellas aportaciones que el Trabajo Social realiza a la función directiva.

\section{PALABRAS CLAVE}

Organizaciones de Servicios Sociales, dirección, supervisión, coordinación, equipo, trabajo en equipo, funciones, capacidades, actitudes, aptitudes y conocimientos del director. 


\section{EL CONTEXTO DE LA REFLEXIÓN}

\subsection{El contexto conceptual.}

El repaso acerca del papel que el trabajador social realiza o puede desarrollar en la dirección de organizaciones prestadoras de Servicios Sociales, ha de partir necesariamente de la identificación de los principales elementos que incluye tal ejercicio.

Consideramos que la dirección de un Centro de Servicios Sociales básicamente consiste en la supervisión y coordinación de los proyectos, objetivos, actividades, técnicas utilizadas y resultados obtenidos por un equipo de trabajo en el desarrollo de las competencias que le han sido encomendadas, y del cual el director es un miembro más con funciones diferenciadas.

Podemos definir el elemento coordinador ${ }^{\prime}$ como la disposición armónica, funcional y ordenada de un grupo para dar unidad de acción, con vistas a conseguir un objetivo común.

La supervisión, con A. Porcel ${ }^{2}$, la entendemos como un proceso continuo de seguimiento, de análisis, de control y de reflexión, que se hace desde una organización, sobre la intervención social que realizan unos profesionales como agentes de una política social, con la ayuda de un supervisor y un equipo de trabajo.

El equipo de trabajo en Servicios Sociales actúa como una instancia complementaria de supervisión de los profesionales individualmente. El supervisor desarrolla sus funciones características con uno y con los otros.

Es evidente que el hablar de las tareas de supervisión y coordinación que ha de desarrollar un director queda falto de contenido si no se hace referencia al grupo de profesionales del que forma parte y al que denominamos "equipo". Este se diferencia de otros grupos de individuos por utilizar, en un momento determinado, un método de trabajo específico, el "trabajo en equipo"3. Este método de trabajo se caracteriza por acumular necesariamente las condiciones que siguen: 1. Existe trabajo en equipo cuando un grupo define un/unos objeti$\mathrm{vo} / \mathrm{s}$ concretos de producción.

Proyecto de coordinación sanitaria entre Atención Primaria y Atención Hospitalaria. Cabueñes (Asturias).

2 PORCEL MUNDO, A., "La supervisión en Servicios Sociales", en Revista de Servicios Sociales y Política Social n. ${ }^{\circ 25}$. Consejo General de Colegios Oficiales de Trabajadores Sociales y Asistentes Sociales. Madrid, 1992. p. 117.

3 PLENCHETTE-BRISSONNET, C., Mètode de Treball en Equip. Ed Pòttic / INTRESS. Barcelona, 1987. pp. 10-14. 
2. Existe trabajo en equipo cuando la naturaleza de esta producción es compatible con objetivos específicos del trabajo en equipo, como son el ahorro de tiempo y energía, la pluralidad de las respuestas y de las intervenciones, el mejor uso de las competencias individuales, el distanciamiento intelectual respecto a lo cotidiano, el análisis sistemático y la investigación, etc.

Existe trabajo en equipo cuando el grupo de individuos elabora "normas de trabajo" constitutivas de un marco metodológico que permita alcanzar el objetivo o los objetivos fijados. Esta condición para el trabajo en equipo nos remite a la idea de que los miembros del grupo han de compartir un común Esquema Conceptual Referencial Operativo (E.C.R.O. $)^{4}$. En las organizaciones de Servicios Sociales se hace imprescindible tener un conjunto de elementos (valores, conceptos, modelos, técnicas e instrumentos) para el abordaje profesional de la realidad que lleguen a ser comunes a todos los miembros del equipo, que se deben consensuar y crear conjuntamente a partir de los saberes y características de cada uno de los miembros.

Existe trabajo en equipo cuando la calidad o la naturaleza del trabajo individual resultan modificados por la elaboración del método de trabajo en equipo.

Situamos la dirección y al director en un contexto de trabajo en equipo, frente a visiones más tradicionales de la función directiva basadas en la línea jerárquica y vertical de mando, donde el director es un satélite en órbita entre una alta jefatura solar y un equipo planetario. Creemos que es en el marco de un equipo de trabajo donde la dirección cobra gran parte de su sentido y donde más ampliamente se pueden desarrollar las funciones que la caracterizan.

\subsection{El contexto organizacional.}

Para situarnos en la globalidad de la reflexión acerca del papel del trabajador social en la dirección de organizaciones y grupos humanos en el ámbito de los SS.SS., es preciso caracterizar brevemente este ámbito y estas organizaciones.

4 Término acuñado por el psicólogo social E. PICHÓN RIVIERE, Asamblea de evaluación institucional. Escuela de Psicología Social. Buenos Aires, 1972. Citado por GEREZ, M., "Contribuciones para una Formulación del Trabajo Social como Disciplina Científica en América Latina", en Secciones del Servicio Social. Ed. Humanitas. Buenos Aires, 1973. pp. $20 \mathrm{yss}$. 


\subsubsection{Los Servicios Sociales.}

No es el objeto de este trabajo definir amplia y profundamente el ámbito de los Servicios Sociales; por el contrario, nos interesa identificar brevemente sus principales elementos constitutivos.

Los Servicios Sociales son uno de los Sistemas Públicos de Protección Social competente en la cobertura de necesidades específicas 5 .



FIGURA 1. El Sistema de Servicios Sociales.

De este modo, y de forma muy sintética, los elementos que definen a los Servicios Sociales son ${ }^{6}$ :

- Un Sistema de PROTECCIÓN SOCIAL.

- Unas necesidades sociales específicas.

- Unas prestaciones sociales específicas.

- Unos equipamientos propios.

- Una normativa, una planificación y unos presupuestos propios.

5 GARCÍA HERRERO, G., Centros de Servicios Sociales. Conceptualización y desarrollo operativo. Ed. Siglo XXI. Madrid, 1988. pp. 18-21.

- GARCÍA HERRERO, G. y RAMÍREZ NAVARRO, J.M., Los nuevos Servicios Sociales. Síntesis de conceptos y evaluación. Ed. Certeza. Zaragoza, 1992. pp. 11-15. 
En su desarrollo, este Sistema de Protección Social se ha articulado en dos niveles básicos de intervención:

1. Un nivel generalista, con el carácter de atención primaria, que constituye la "puerta de acceso" al Sistema y se dirige a toda la población por necesidades: los Servicios Sociales Generales, Comunitarios o de Atención Primaria.

2. Un nivel especializado, con el carácter de atención secundaria, dirigido a sectores de población con necesidades específicas: los Servicios Sociales Especializados.

\subsubsection{Las Organizaciones de Servicios Sociales.}

La prestación de los Servicios Sociales se materializa a través de diferentes organizaciones sociales. Éstas comparten elementos comunes a toda organización, y también desarrollan componentes que las singularizan?

Amparo Porcel ${ }^{8}$, basándose en los conceptos aportados por Hasenfeld y English", define las organizaciones de Servicios Sociales como "organizaciones al servicio de la persona (OSP)", e identifica tres características básicas del trabajo que han de realizar:

a. Su input son seres humanos con atributos específicos.

b. Su output o "producción" es cambiar la situación de las personas que atienden.

c. Su mandato es ser un servicio, generalmente derivado de un marco legal que define sus competencias.

Junto a estos rasgos básicos, y con base en los trabajos de Peiró y Medina $^{10}$, Porcel ${ }^{11}$ y Bueno Abad ${ }^{12}$, otros elementos terminan por caracterizar organizacionalmente a los Servicios Sociales:

LÓPEZ CÁBANAS, M. y GALLEGO GALLEGO, A., "Análisis organizacional y propuestas de desarrollo en los Servicios Sociales Comunitarios", en III Jornadas de Intervención Social. Tomo I. Ed. INSERSO. Madrid, 1993. pp. 83-84.

\& POCEL, A. y VÁZQUEZ, C., La supervisión. Espacio de aprendizaje significativo. Instrumento para la gestión. Ed. Certeza / INTRESS. Zaragoza, 1995. p. 158.

9 HASENFELD y ENGLISH citados por PORCEL, A., "La intervención del trabajador social en la planificación, gestión y desarrollo de los Servicios Sociales", en Documentación Social $n^{\circ}$ 79. Ed. Cáritas Española. Madrid, 1990.

10 PEIRÓ, J.M. a y MEDINA, M., "Aspectos psicosociales de la gestión de organizaciones de Servicios Sociales", en IV Jornadas de Intervención Social. Tomo 2. Ed. INSERSO. Madrid, 1996. pp. 1148-1157.

11 PORCEL, A. y VÁZQUEZ, C. (1995), op. cit. pp. 158-160.

12 BUENO ABAD, J.R., Servicios Sociales: Planificación y organización. Ed. Mestral Llibres. Valencia, 1988. pp. 73-74. 
1. Organizaciones de servicios.

La producción y la entrega del producto son simultáneos y realizados por la misma persona. El consumo se produce también en el mismo acto, lo que no permite la comprobación previa de la calidad del servicio: el cliente lo demanda basándose en la confianza.

No existen stocks. Ello obliga a una gestión diferente de la producción y la entrega.

El servicio combina, además, componentes técnicos y profesionales con componentes ideológicos.

2. Organizaciones públicas, en su mayoría, y privadas.

La Administración es la promotora y proveedora más importante de Servicios Sociales. Ello implica que el servicio público se enfrente a dos tipos de demandas que han de articularse entre sí convenientemente: las demandas e intereses de los ciudadanos y las del proyecto político del equipo que dirige la organización pública.

Coexistiendo con el sector público, se ha desarrollado en gran medida el sector privado $^{13}$, tanto lucrativo como no lucrativo, a través de fórmulas de gestión mixta de los Servicios Sociales: unas veces complementando los servicios públicos; otras, prestando éstos mediante gestión indirecta (convenios, contrataciones, etc.). Al mismo tiempo, la Administración pública ha generado sistemas de autorización de la actividad privada en el ámbito de los Servicios Sociales, para garantizar la calidad de los servicios y de los derechos de los ciudadanos.

3. La misión de las organizaciones de Servicios Sociales.

Estas organizaciones se diseñan y crean con la misión de cambiar las situaciones de las personas que son atendidas mediante la definición de una serie de objetivos generales y estratégicos. Son posibles múltiples formulaciones de esta misión, cuyo contenido va a implicar componentes ideológicos y políticos, además de los componentes técnicos.

Esta multiplicidad, unida a un grado importante de generalidad y ambigüedad, plantea problemas para la concreción de los fines y objetivos, su priorización y su evaluación.

4. El objeto e instrumento de la producción.

Estas organizaciones trabajan con y en las relaciones interpersonales. Muchos de los procesos que emprenden tienen que ver con cambios de actitudes, con la socialización de los individuos, 
con aspectos biográficos de las personas que intervienen en estos procesos y entran en juego valores, ideas, actitudes y hábitos.

5. La tecnología.

La tecnología de las organizaciones de Servicios Sociales no es estándar, ya que está basada en las relaciones interpersonales, que comportan incertidumbre e impredecibilidad sobre el estado del cliente con quien se trabaja.

6. Los profesionales.

Las organizaciones al servicio de la persona emplean profesionales cualificados. Esto es debido a la naturaleza y complejidad de los problemas humanos a que estas organizaciones pretenden dar respuesta. El tipo de trabajo que se requiere es "creativo" y "artesanal" y es complejo.

7. La eficacia y la eficiencia.

La medida de la eficacia (grado de consecución de objetivos) y de la eficiencia (consecución de los objetivos con los resultados adecuados y los menores costes posibles) es compleja en este tipo de organizaciones, por cuanto no puede seguir exclusivamente criterios economicistas. Esta evaluación se complica aún más si consideramos la dificultad para establecer objetivos precisos, la estrecha relación entre la gestión y las decisiones políticas, la dificultad para medir los impactos producidos por determinados programas o proyectos, etc.

Este conjunto de características propias de las organizaciones de Servicios Sociales actúan como elementos definitorios y condicionantes del tipo de estructura organizativa a desarrollar, de la tecnología a utilizar y del modelo directivo y de las estrategias de gestión necesarias para el funcionamiento organizacional.

\subsubsection{Los Centros de Servicios Sociales: estructura, equipo y territorio.}

Participando de las características señaladas para las organizaciones de Servicios Sociales, los denominados "Centros Sociales" o "Centros de Servicios Sociales", desde los Servicios Sociales Generales, son los responsables de la implantación y desarrollo de los programas de Prestaciones Básicas de Servicios Sociales y son la puerta de acceso a la red pública de Servicios Sociales.

De este modo, los Centros Sociales se desarrollan y configuran sobre la base de tres grandes ejes:

- Una estructura integrada y descentralizada de ámbito comunitario, es decir, un lugar de referencia de programas y proyectos, servicios, prestaciones y equipamientos. 
- Un equipo de trabajo, constituido por profesionales de distintas disciplinas con tendencia al trabajo interdisciplinar.

- Un territorio, diana de los programas de Servicios Sociales, que define y limita geográfica y poblacionalmente el ámbito de la intervención del Centro Social.

\section{LA DIRECCIÓN DE UN CENTRO DE SERVICIOS SOCIALES}

\subsection{Pero ¿Por qué...?. TRADICIÓN versus INNOVACIÓN.}

El Centro Social constituye una organización compleja en la que se hace necesario garantizar un desarrollo armónico de funciones y recursos diferentes a través de un profesional con funciones también diferenciadas que asume la dirección del Centro Social.

Hemos de recordar que toda la organización debe ser una cadena de valor añadido, de modo que cada una de las actividades que en ella se realizan debe añadir valor, ya que las tareas o funciones que no añaden valor lo que hacen es añadir coste. Descartamos aquí una figura directiva tradicional que únicamente actúa como enlace informativo y de mando entre el Centro de SS.SS. y su entorno (organizaciones, comunidad, etc.). Optamos, sin embargo, por un modelo de dirección con parcelas propias de decisión; que realiza aportaciones claras con relación a los productos, servicios y actuaciones del Centro Social; que facilita y apoya en su ámbito funcional el trabajo de los profesionales y el funcionamiento del Centro Social y que se orienta hacia la motivación del equipo, al logro de resultados y el fomento de la creatividad e innovación; en definitiva, una figura que actúa estimulando el funcionamiento global del Centro Social.

Entendemos, con G. García Herrero ${ }^{14}$, que el Director no se ha de limitar a ser un superior jerárquico en la estructura, preocupado en exclusiva por el mantenimiento de las normas que le afecten. Por el contrario, éste es el responsable de organizar en la forma más racional (minimizando costes y maximizando resultados) los recursos de los que el Centro dispone, para conseguir con la mayor eficacia y eficiencia posible, los objetivos y metas que esta estructura integrada tiene encomendados.

Por ello, proponemos la superación de la gestión tradicional en la dirección de Centros de Servicios Sociales para dar paso a un modelo más innovador. En esta línea de ideas, es conveniente que pongamos 
en relación los aspectos diferenciales de la gestión tradicional y de la gestión innovadora:

\begin{tabular}{|c|c|c|c|}
\hline & & Gestión tradicional & Gestión innovadora \\
\hline \multirow{3}{*}{ Criterios } & $\begin{array}{l}\text { Objetivos } \\
\text { del puesto }\end{array}$ & $\begin{array}{l}\text { Gestión de los recursos } \\
\text { disponibles }\end{array}$ & $\begin{array}{l}\text { Creación de nuevos } \\
\text { recursos }\end{array}$ \\
\hline & $\begin{array}{c}\text { Principios de } \\
\text { dirección } \\
\text { (Management) }\end{array}$ & $\begin{array}{c}\text { Principios universales } \\
\text { de gestión }\end{array}$ & $\begin{array}{c}\text { Dirección situacional } \\
\text { Flexibilidad }\end{array}$ \\
\hline & $\begin{array}{c}\text { Procedimientos } \\
\text { de trabajo }\end{array}$ & $\begin{array}{l}\text { Orientados a actividades } \\
\text { aisladas }\end{array}$ & $\begin{array}{l}\text { Orientados a procesos } \\
\text { integrados }\end{array}$ \\
\hline de & $\begin{array}{l}\text { Criterios de } \\
\text { evaluación }\end{array}$ & $\begin{array}{c}\text { Eficacia } \\
\text { (relac. producción /tiempo) }\end{array}$ & $\begin{array}{c}\text { Eficiencia } \\
\text { (relac. coste /beneficio) }\end{array}$ \\
\hline \multirow{4}{*}{ del } & $\begin{array}{l}\text { Orientación } \\
\text { al mercado }\end{array}$ & $\begin{array}{l}\text { Vende productos o } \\
\text { servicios estándar }\end{array}$ & $\begin{array}{l}\text { Satisface necesidades } \\
\text { de la clientela }\end{array}$ \\
\hline & $\begin{array}{c}\text { Formación } \\
\text { personal }\end{array}$ & Aprendizaje ocasional & $\begin{array}{l}\text { Formación permanente } \\
\text { Descubrimiento personal }\end{array}$ \\
\hline & $\begin{array}{l}\text { Estilo de } \\
\text { mando }\end{array}$ & $\begin{array}{l}\text { Obediencia basada } \\
\text { en el status }\end{array}$ & $\begin{array}{l}\text { Adhesión } \\
\text { Trabajo en equipo }\end{array}$ \\
\hline & Incentivos & $\begin{array}{l}\text { Incentivos extrínsecos } \\
\text { al trabajo }\end{array}$ & $\begin{array}{l}\text { Incentivos intrínsecos } \\
\text { Enriquecimiento de tareas }\end{array}$ \\
\hline
\end{tabular}

FIGURA 2. Gestión Tradicional versus Gestión Innovadora.

Además, hoy las tareas del director en la organización cobran especial relevancia en el modelo mixto de gestión extendido en los Servicios Sociales ${ }^{15}$, en virtud del cual se establece un sistema de corresponsabilidad entre iniciativa pública, privada y social. En este modelo, la Administración pública incorpora al sector privado en los servicios públicos, a través del convenio o el concierto, para la prestación de servicios (Servicio de Ayuda a Domicilio, Programas de Intervención Familiar, Programas de Atención a Colectivos o Necesidades específicas, etc.); y se obliga, entre otras, a realizar una función de inspección y evaluación de los servicios prestados. $\mathrm{Y}$ es al director, desde la estructura descentralizada y cercana al ciudadano que es el Centro Social, a quien le es atribuida esta función de inspección y evaluación en su ámbito territorial.

15 García RoCA, J., Público y Privado en la Acción Social. Del Estado del Bienestar al Estado Social. Ed. Popular. Madrid, 1992. pp. 46, 117-126, 157-161.

GARCÍA HERRERO, G. y RAMÍREZ NAVARRO, J.M. (1992), op. cit. pp. 83-93.

BUENO ABAD, J.R. (1988), op. cit. pp. 81-84. 


\subsection{Funciones y roles del director de un Centro de Servicios Sociales}

Por lo general, los Centros Sociales, por su carácter descentralizado, dependen orgánicamente de las Administraciones locales (Ayuntamientos, Diputaciones o entidades supramunicipales). El director del Centro Social, siguiendo la terminología empleada por Mintzberg ${ }^{16}$, se sitúa en la línea de mando o dirección intermedia, entre el vértice estratégico (Concejal, Diputado, etc.) y el núcleo de operaciones (el personal asignado al Centro Social y a sus unidades dependientes), junto a otros mandos medios de la organización (responsables de Programas Centrales, personal del staff, etc.).

Las funciones de la dirección y los roles que ha de asumir en el ejercicio de aquéllas son, con carácter general, los que la gestión de toda organización implica y son una combinatoria de las mismas funciones organizacionales. Peiró y Medina han elaborado una interesante síntesis de esta combinación de funciones, donde hemos señalado las relaciones que mantienen:

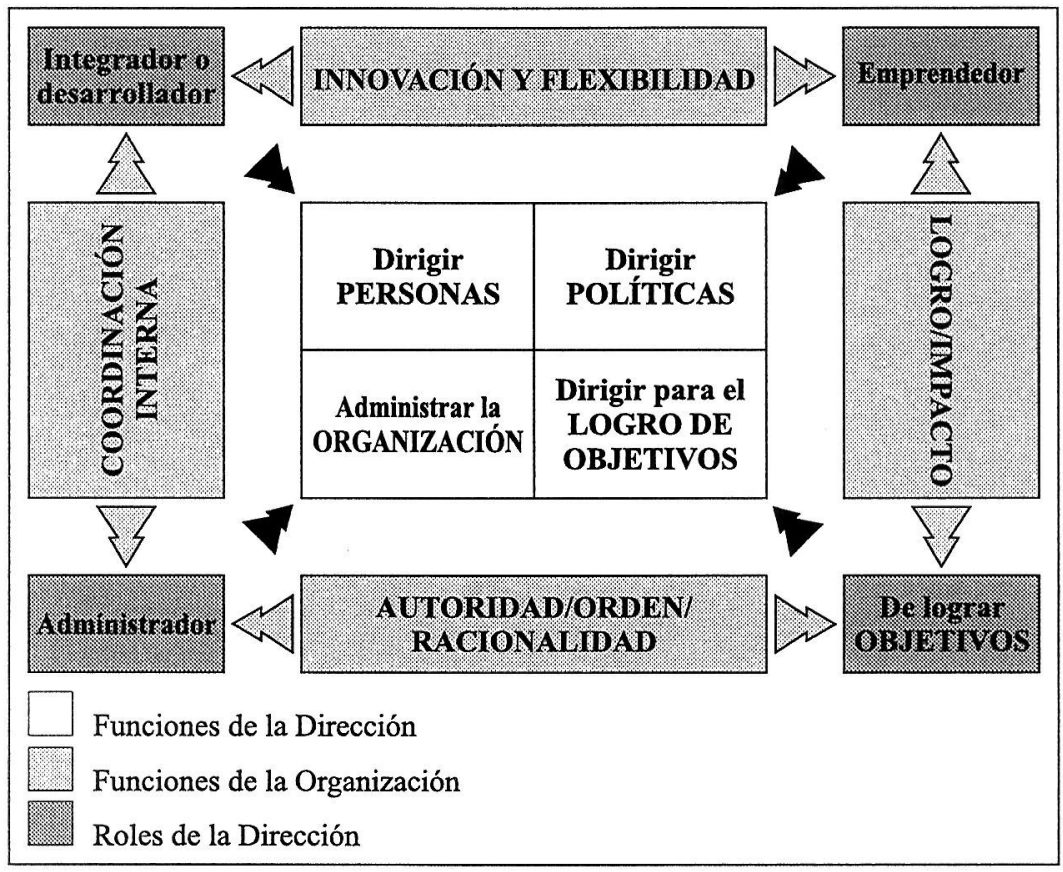

FIGURA 3. Funciones organizacionales y funciones y roles de la Dirección.

16 MINTZBERG, Henry, La estructuración de las organizaciones. Ed. Ariel. Barcelona, 1995. pp. 52-55. 
En virtud de la posición intermedia que ocupa en la organización, le es atribuida una de las funciones vitales para el logro de la misión que la organización tiene encomendada: la función de dirección o "pilotaje", que incluye ${ }^{17}$ :

a. Dirección operacional: desarrollo de las actividades definidas por el ápice estratégico para su unidad y territorio.

b. Enlace vertical: transmisión de la planificación hacia abajo, hacia arriba transmite información para el seguimiento, el control y la evaluación.

c. Enlace horizontal: mantiene la coordinación precisa dentro de la organización con otras unidades y servicios y con el entorno.

d. Mando y dirección de personas, instrucción y motivación.

A. Porcel ${ }^{18}$ ha desarrollado el contenido de esta función partiendo de los trabajos de Mintzberg ${ }^{19}$ y Kadushin ${ }^{20}$.

\subsubsection{Función de dirección operacional.}

Esta función reproduce las actividades del ápice estratégico o alta dirección, pero en el ámbito del Centro Social. Se compone de varias subfunciones:

1. De prospectiva: consiste en conocer la evolución y tendencias tanto del entorno interior (zona, tipo de población, necesidades, demandas, recursos, etc.) como exterior (la organización, otros servicios o departamentos).

2. De relaciones internas y externas: desarrollo de estrategias para mantener el equilibrio en el entorno interior y el clima de colaboración con el exterior.

3. De planificación: pasa por desarrollar la planificación general de la organización en su ámbito territorial.

\subsubsection{Función de dirección de personas.}

Tiene como finalidad guiar los trabajos operacionales, tomando las decisiones oportunas sobre:

- Cuándo y en qué cantidad debe hacerse el trabajo.

- Cómo y en qué condiciones va o está yendo la realización.

- Qué dificultades surgen y qué cambios se precisan.

Es una de las funciones fundamentales para la correcta realización de la producción, y entronca directamente con el uso de la supervisión

17 PORCEL, A. y VÁZQUEZ, C. (1995), op. cit. pp. 135-138.

Is PORCEL, A. y VÁZQUEZ, C. (1995), op. cit. pp. 163-176.

19 MINTZBERG, Henry (1995), op. cit.

20 KADUSHIN, A. Supervision i social work. Columbia University Press. New York, 1985. 
en la dirección de Centros Sociales, sobre la base del necesario soporte que el director ha de prestar a los profesionales.

Dentro de esta gran función, se pueden identificar varias subfunciones del director-supervisor ${ }^{21}$ :

\section{Directiva/Administrativa.}

Consiste en asegurar que el trabajo se lleve a cabo de acuerdo con el encargo. Su objetivo principal es asegurar la coherencia entre planificación y procedimientos.

Incluye las tareas de:

a. Reclutamiento y selección de personal. Esta función en la Administración pública española no es realizada directamente por la dirección del Centro Social. En su lugar, se define el perfil de los puestos necesarios y el de los candidatos, y el número de trabajadores necesario.

b. Iniciación y ubicación del nuevo trabajador.

c. Planificación y asignación del trabajo.

d. Delegación del trabajo.

e. Seguimiento, revisión y evaluación del trabajo asignado y/o delegado.

2. Educativa/Capacitadora.

Es, con la función de apoyo, la característica que diferencia este modelo de dirección de otros. Consiste en dar respuesta a las necesidades de conocimiento del trabajador, de entendimiento conceptual y de desarrollo técnico. Unas veces la respuesta será directa por parte del supervisor, otras indirecta a través de la orientación hacia la formación específica del Trabajador Social. Su objetivo es subsanar las lagunas de conocimiento y mejorar las habilidades.

Complementa la supervisión administrativa ya que ayuda al profesional a comprender cómo ha de realizar las tareas y cómo hacerlas mejor.

3. Apoyo.

Constituye el reconocimiento de que los profesionales de las organizaciones de Servicios Sociales, y especialmente los Trabajadores Sociales, tienen un mayor número de demandas que superan sus recursos personales y emocionales que el que tienen otras profesiones. Tiene que ver con el estado de ánimo de los trabajadores y la satisfacción con relación a la tarea. Su objetivo para el supervisor consiste en ayudar a los supervisados a superar el estrés

21 NICHOLS, J. y CAVE, C., "Supervisión: Algunos principios y consideraciones para la práctica", en Revista de Servicios Sociales y Política Social n. ${ }^{\circ} 25$. Consejo General de Colegios Oficiales de Trabajadores Sociales y Asistentes Sociales. Madrid, 1992. pp. 7-9. 
y la ansiedad inherentes al desempeño del trabajo social e incrementar la moral y la satisfacción en el puesto de trabajo.

4. Evaluadora.

Consiste en el control de la eficacia de la práctica del trabajador utilizando el «feedback» como un medio de apoyo a su desarrollo profesional y para asegurar que el cliente obtenga «el mejor servicio posible». Pasa por la revisión continuada del proceso de trabajo, que ayude a pensarlo y a repensarlo constantemente, valorando los logros, las desviaciones, los imprevistos y facilitando la toma de decisiones.

Como es fácil advertir, estas funciones habitualmente se presentan combinadas en el espacio de la dirección-supervisión, con mayor énfasis en unas o en otras, según las necesidades que se pretende cubrir en cada momento.

\subsubsection{Función de coordinación.}

Las organizaciones de Servicios Sociales, en su calidad de sistemas abiertos, reciben e intercambian información propia y del entorno en un proceso de retroalimentación ${ }^{22}$.

Es función del director posibilitar la conexión vertical y horizontal, la coordinación y la comunicación:

- Entre los miembros del equipo. Ello unifica los esfuerzos de los diferentes trabajadores hacia el logro de los objetivos de la organización.

- Con otras unidades de la organización.

- Con otras organizaciones de Servicios Sociales.

La posición del mando medio en la estructura de la organización es un lugar clave para la recogida, procesamiento y distribución de la información, tanto en sentido ascendente (necesidades y demandas, dificultades de ejecución del trabajo, quejas, etc.), como descendente (directrices, formas de trabajo, etc.) entre, por un lado, el núcleo de operaciones y la población, y, por otro, la alta dirección.

En síntesis, en un Centro de Servicios Sociales, al igual que ocurre en las funciones que ejercen las redes sociales ${ }^{23} \mathrm{o}$ en la intervención profesional con familias, corresponde al director ejercer funciones de dos tipos principalmente ${ }^{24}$ : la intervención comunitaria", en Intervención Psicosocial Vol II N*4. Madrid 1993. pp. 72-73.

24 PLENCHETTE-BRISSONNET, C. (1987), op. cit. p. 68. 
a. Funciones de APOYO a los profesionales, orientadas a servir de soporte al trabajo de los miembros del equipo y a resolver las dificultades que de éste se derivan.

b. Funciones de CONTROL de los profesionales, orientadas a vigilar las tareas, actividades, proyectos, etc., para garantizar el logro de los objetivos marcados por el equipo y por la organización.

La organización describe y limita estas funciones directivas generales a través de una definición formal del puesto del directivo intermedio, que da respuesta a sus necesidades de funcionamiento. Junto a ello, encontramos que los miembros de la organización en general, y del equipo de trabajo en particular, someten a la función directiva a un nuevo modelaje, de carácter más específico y detallado que el anterior. Con ello, el equipo y sus integrantes pretenden para dar respuesta a sus necesidades como individuos, como profesionales y como grupo, configurando una definición informal del puesto, dentro de los límites formales atribuidos a éste.

\subsection{Características del director.}

El director de un Centro de Servicios Sociales debería identificarse y actuar como el líder natural del grupo de trabajo al cual pertenece, en calidad de lo cual es limitado en el cumplimiento de sus funciones y le es exigido en mayor medida que al resto de miembros del grupo. Esto no siempre ocurre en las organizaciones de Servicios Sociales. En ellas el liderazgo de la Dirección suele ser formal, por cuanto el director ha sido nombrado o ha accedido al puesto desde el exterior, y es habitual que sea recibido y tratado como miembro ajeno al grupo.

En la realidad organizativa en que nos situamos, no se puede dirigir el esfuerzo de las personas con el mando, ordeno y controlo tradicional. Por el contrario, es necesario un tipo de dirección que en lugar de estar basado en el poder y el rango, lo esté en el respeto y la confianza que el directivo ha de ganarse de los trabajadores. Y por ello, la persona destinada en este puesto ha de reunir unas características específicas y precisa una adecuada formación en management, donde los aspectos humanos son tan importantes como los técnicos.

En los procedimientos actuales de acceso a la Administración pública española no siempre se priman aspectos de vital importancia en el perfil de un director de un Centro de Servicios Sociales, como son el buen ejercicio de la práctica, el conocimiento profundo de los Servicios Sociales (en su teoría y en su práctica), las habilidades necesarias para la dirección de Recursos Humanos y las aptitudes/actitudes personales en relación con el trabajo con personas y con el trabajo en equipo. 
Además, por su situación en la estructura de la organización, el director se encuentra en una posición difícil al situarse en un nivel medio entre la alta dirección y los trabajadores (efecto cartílago). Es por ello, que la dirección requiere una habilidad social considerable además de conocimientos y habilidades técnicas que refuercen la autoridad formal que la organización le atribuye.

Las capacidades, aptitudes, actitudes y conocimientos que consideramos necesarios en el director y que a continuación se relacionan determinan en sí mismos un modelo de dirección.

\subsubsection{Capacidades.}

Para desarrollar las funciones que la organización les asigna, los directores recurren a cuatro tipos de capacidades de administración ${ }^{25}$.

1. Capacidades técnicas.

Son las que permiten dominar los procesos de trabajo, los métodos de producción.

Dado que las tecnologías de las organizaciones de Servicios Sociales no son estandarizables y que puede haber distintos enfoques y formas de intervenir para conseguir resultados similares, el director ha de ser flexible en cuanto a aceptar formulaciones técnicas diferentes por parte del equipo. Para ello, ha de conocer más de un enfoque para así valorar adecuadamente la idoneidad de cada uno con relación al tipo de intervención necesaria.

Incluye también las capacidades para el dominio de técnicas de análisis y estudio de las demandas del entorno, y otras referidas a la producción del grupo ${ }^{26}$.

2. Capacidades administrativas.

Son las que sirven para que todas las partes del servicio encajen unas con otras. Permiten formular objetivos, orientar al equipo hacia la eficacia, facilitar el trabajo en equipo, etc. En definitiva, proporcionan procedimientos claramente estructurados y ofrecen un feedback positivo a los trabajadores sobre sus relaciones con el servicio y sobre su práctica.

3. Capacidades conceptuales.

Son las que se refieren a la capacidad de síntesis, de inferir y de deducir elementos útiles para la intervención a partir de situaciones, hechos y resultados anteriores.

25 HODGETTS, Richard M., El supervisor eficiente. Un enfoque práctico. Ed. Mc Graw Hill. México, 1987, citado en PORCEL, A. y VÁZQUEZ, C. Op. cit. pp. 211-213.

36 Estas técnicas de apoyo a la producción del grupo han sido identificadas por PLENCHETTEBRISSONNET, C. (1987), op. cit. pp. 68-75. 


\section{Capacidades humanas.}

Se basan en las habilidades de comunicación y de relaciones interpersonales. Tienen que ver con su capacidad de liderazgo, con sus habilidades sociales y con su capacidad de empatía. Incluyen:

- Capacidad de escucha.

- Capacidad de comunicación completa y libre con el equipo de trabajo.

- Actuar como facilitador y como línea de comunicación entre la línea operativa y la alta dirección.

\subsubsection{Actitudes}

Estas actitudes orientarán y darán contenido a la actuación del director-supervisor, y definirán el uso de unas técnicas en lugar de otras en el proceso directivo ${ }^{27}$ :

- Confianza y aceptación hacia los profesionales del equipo, a fin de optimizar la autonomía y la facultad de juicio propio y crítico del supervisado.

- Empatía.

- Respeto frente a las opiniones y forma de trabajar de los demás.

- Disponibilidad para consultas de carácter profesional, e incluso personal.

- Compartir conocimientos y enseñar con su práctica y con sus orientaciones.

- Consenso y cooperación para la solución de problemas derivados de la participación democrática más que de los enfoques jerárquicos centralistas.

- Aprender y crecer a partir de la experiencia proporcionada por el trabajo.

- Establecer una relación de liderazgo consultivo más que una relación de subordinación.

\subsubsection{Aptitudes.}

Las aptitudes que recogemos actúan como factores de éxito en el modelo de dirección-supervisión que planteamos. Se basan en las formuladas por Richard E. Boyatzis ${ }^{28}$, Amparo Porcel ${ }^{29}$ y Claudine Plenchette-Brissonnet ${ }^{30}$, incluyendo algunas propuestas del autor.

27 PORCEL, A. y VÁZQUEZ, C. (1995), op. cit. pp. 209.

28 BOYATZIS, Richard E., The competens Manager. Ed. Wiley. New York, 1982, citado en PORCEL, A. y VÁZQUEZ, C. (1985), op. cit. pp. 214-215.

29) PORCEL, A. y VÁZQUEZ, C. (1995), op. cit. p. 215.

30 PLENCHETTE-BRISSONNET, C. (1987), op. cit. pp. 68-74. 
1. Alta capacidad de EXIGENCIA. Un grupo humano sin un líder que practique la exigencia hacia sí mismo y hacia los demás, acabará convirtiéndose en un grupo de incompetentes o en un asilo de burócratas, que sólo trabajan para su propia comodidad y beneficio.

2. CONFIANZA interna y externa y DOMINIO DE SÍ MISMO. Es fundamental que el director tenga confianza en sí mismo, en sus habilidades y en sus conocimientos. Al mismo tiempo, ha de generar confianza en sus colaboradores para optimizar su autonomía y la facultad de juicio propio del trabajador.

La persona con confianza y dominio de sí mismo tiende a aplicarse el concepto de coste-beneficio a sí mismo, al grupo de trabajo y a la organización en general, antes que expresarse o actuar sobre la base de sus necesidades o deseos personales.

3. VISIÓN más amplia y profunda que el resto de trabajadores para:

- Conducir a metas más exigentes.

- Ofrecer seguridad y mover a innovar.

- Romper con la rutina.

4. EMPRENDEDOR. Consiste en analizar constantemente la manera de aprovechar todas las oportunidades que ofrece el cambio, minimizando los efectos de los problemas.

5. FORMADOR. Para lograr mayores objetivos es necesario un mayor grado de formación teórico-práctica, unos conocimientos superiores y una mejora permanente de habilidades.

6. CONTROL POSITIVO. Se trata de un control preventivo o anticipativo que establece parámetros de control antes de que aparezcan los errores.

7. OBJETIVIDAD. Es la habilidad para mantenerse relativamente ecuánime sin dejarse influir por excesivos prejuicios o perspectivas personales. Este factor facilita en mayor medida la consideración de los puntos de vista de los demás.

8. FRANQUEZA. Los supervisores eficientes demuestran tener un alto grado de espontaneidad o habilidad para expresar con libertad, facilidad y seguridad sus sentimientos y emociones.

9. Utilización del PODER INDIVIDUAL. Consiste en el uso de la influencia para obtener obediencia.

10.Utilización del PODER DEL GRUPO. Es el uso de las formas de influencia como las alianzas, las redes, las coaliciones y los equipos para llevar a cabo el trabajo. Las personas que usan el poder socializado tienden a sentirse miembros de un equipo o grupo.

11.MODELAJE de la actuación. Los supervisores acostumbran a su- 
pervisar de la misma forma como ellos son supervisados; y de la misma forma que ellos traten a los miembros del equipo, así actuará el equipo.

12. ACTUAR para integrar las necesidades de producción del servicio con las necesidades socioemocionales de los trabajadores, equilibrando los objetivos del Centro de Servicios Sociales con el ánimo de los profesionales, haciendo las demandas de tareas de acuerdo con los intereses de los miembros del grupo, equilibrando tareas más administrativas e instrumentales con otras más conceptuales, creativas, etc.

13.Ser ANIMADOR DEL EQUIPO. Actuar como garante de la consecución de los objetivos del equipo. Esta aptitud será la que conformará su papel y caracterizará su tarea, sobre todo en las reuniones de equipo.

\subsubsection{Conocimientos.}

El primer conocimiento de este mando medio es el del MANAGEMENT, el de la dirección orientada hacia el trabajo bien hecho y hacia el apoyo a los trabajadores, preocupado por su propia formación permanente y la del equipo de trabajo, y que actúa de forma altamente motivante.

1. Técnicas de MOTIVACIÓN.

Tan importante es motivar a los trabajadores como eliminar los elementos desmotivadores que existen en el área de trabajo.

2. Técnicas de DELEGACIÓN.

La delegación es un instrumento importante para practicar la formación en el puesto de trabajo y para estimular el crecimiento en conocimientos y en responsabilidad de los trabajadores. También es necesaria para eliminar de la línea directiva trabajos de otros niveles que son necesarios, con el fin de disponer del tiempo preciso para las tareas directivas propiamente dichas.

3. Técnicas de GENERACIÓN DE OBJETIVOS en grupo.

4. Técnicas de TRATAMIENTO DE LA INFORMACIÓN.

El director debe dominar la INFORMACIÓN, es decir, la capacidad de sintetizar, extraer y hacer visibles los datos más importantes cuyo análisis servirá de soporte para la toma de decisiones, distinguiendo especialmente la información más útil para el control anticipativo del trabajo.

5. Conocimientos globales de la ORGANIZACIÓN.

Debe poseer un conocimiento general de la organización, y una comprensión global de todas las funciones que se desarrollan en la 
misma, con el fin de facilitar la integración del Centro de trabajo en los objetivos generales de la organización.

6. Conocimientos de las TECNOLOGÍAS de su área de trabajo.

Ha de poseer un buen conocimiento de todas las tecnologías que se precisan en su centro de trabajo. Ello no impide que en algún aspecto puntual y específico, otro trabajador tenga mayores conocimientos.

7. Técnicas de ORGANIZACIÓN MODERNA y de PRODUCTIVIDAD.

Con el fin de aumentar la productividad y los resultados, el director se verá obligado a optimizar constantemente los procesos que dirige. Para ello necesita conocer las técnicas modernas de organización, los sistemas de análisis de tiempos y métodos, así como la racionalización de los procesos. Además, precisa explorar nuevos sistemas de recompensas, reconocimiento profesional y desarrollo profesional.

8. APRENDER a aprender.

Aprender constantemente. Reaprender aspectos olvidados o mal aprendidos. Desaprender el trabajo rutinario, las decisiones basadas sólo en la experiencia, los modos de actuar de la organización jerárquica piramidal y el trabajo individual frente al trabajo en equipo.

\section{III.APORTACIONES DESDE EL TRABAJO SOCIAL A LA DIRECCIÓN DE ORGANIZACIONES Y EQUTPOS DE TRABAJO}

El Trabajo Social como disciplina y como profesión ${ }^{31}$ aporta a la dirección de organizaciones y de equipos de trabajo un sistema integral de abordaje de la realidad, en este caso, de la realidad de la organización, en el que destacamos:

a. El apoyo de una Teoría sistematizada en el ejercicio de la profesión $^{32}$.

b. El uso de un Método sistemático de trabajo.

c. La orientación de sus actuaciones según un Código Internacional de Deontología Profesional ${ }^{33}$, que recoge los principios éticos bá-

31 Estta doble dimensión del Trabajo Social es planteada por BÁÑEZ TELLO, T., "Situación profesional a la que nos enfrentamos hoy", en DI. Aportes desde el Trabajo Social. N." 4/5 (Actas de las Jornadas de Trabajo Social 1995). Colegio Oficial de Diplomados en Trabajo Social y Asistentes Sociales. Alicante, 1995. p. 85.

32 Este elemento y la existencia de un código de ética son esñalados entre los atributos esenciales de una profesión por GREENWOOD, E. (1996), Atributes of a profession.

33. Aprobado por la Asamblea de la Federación Internacional de Trabajadores Sociales en julio 
sicos de la profesión, recomienda procedimientos en situaciones de dilemas éticos, y aborda la ética en las relaciones derivadas del ejercicio de la profesión.

d. Su vinculación y dependencia respecto a los Modelos de Política Social en cuyo marco los trabajadores sociales ejercen su activi$\mathrm{dad}^{34}$.

Acostumbramos a referirnos a este sistema de acercamiento, conocimiento e intervención con la realidad sólo desde la perspectiva de las intervenciones directas que desde las organizaciones de bienestar social realizan los trabajadores sociales con individuos, familias y/o grupos. Sin embargo, este sistema también conserva su vigencia si consideramos la intervención indirecta que realizan los trabajadores sociales a través de funciones gerenciales o de dirección y supervisión de profesionales y organizaciones ${ }^{35}$.

Como aspectos más destacados de este sistema podemos señalar los que siguen y lo singularizan:

- Se basa en el trabajo con personas con necesidades y conflictos, individual o grupalmente considerados. En esta consideración se incluyen el equipo de trabajo y sus miembros.

- Mantiene una actitud de respeto a los valores, ideologías, conductas, etc., de los miembros del equipo de trabajo.

- Sigue criterios de globalidad en el análisis y abordaje de los problemas y necesidades de una organización, puesto que éstos forman parte de una dinámica compleja que ha de considerarse de manera conjunta.

- Pretende la integración de programas, servicios, actividades, profesionales y equipamientos en un todo coordinado que es la organización, y en nuestro caso, el Centro Social.

- Con visión anticipatoria frente a problemas y necesidades de los grupos humanos y la organización, actúa para la prevención de conflictos y dificultades internas y con el entorno.

de 1994 y donde se integran, revisados, documentos anteriores de 1976 y 1990: "La Etica del Trabajo Social: Principios y Criterios". F.I.T.S. Oslo, 1994.

i4 ROSSELL, T., "La formación para el Trabajo Social". VII Congreso Estatal de Trabajadores Sociales y Asistentes Sociales. Barcelona, 1992. Citada por BÁÑEZ TELLO, T. "La formación en el Trabajo Social", en Revista de Servicios Sociales y Política Social N. 30 . Consejo General de Colegios Oficiales de Trabajadores Sociales y Asistentes Sociales. Madrid, 1993. p. 150 .

35 Con relación a la intervención directa / indirecta, nos remitimos al "Dictamen sobre definicion de objetivos y funciones de profesionales" elaborado por el Consejo General de Colegios Oficiales de Trabajadores Sociales y Asistentes Sociales y publicado en la Revistu de Servicios Sociales y Politica Social N. ${ }^{\circ} 30$. Consejo General de Colegios Oficiales de Trabajadores Sociales y Asistentes Sociales. Madrid, 1993. pp. 9-11. 
- Fomenta la participación del equipo y sus miembros en la toma de decisiones y en la resolución de conflictos.

- Se orienta hacia las potencialidades/capacidades de los trabajadores. El arte de la dirección pasa por saber motivar e impulsar a los trabajadores al ejercicio de sus potencialidades profesionales en el contexto organizacional. Este espacio de crecimiento es la supervisión y la formación continuada.

- Por último, se orienta hacia la autonomía de los profesionales. El director actúa como motor y como apoyo para el trabajo autónomo de los profesionales en la organización. Para ello es fundamental el incremento de la confianza recíproca y la elevación del nivel de delegación de tareas hacia los trabajadores como consecuencia directa de la propia labor directiva. Esto no significa el «abandono del profesional», sino el acompañamiento continuo pero oculto y la actuación sólo cuando lo demande el profesional o las decisiones exceden de su ámbito competencial.

\section{BIBLIOGRAFÍA}

BÁÑEZ TELLO, T. "La formación en el Trabajo Social", en Revista de Servicios Sociales y Política Social $n^{\circ} 30$. Consejo General de Colegios Oficiales de Trabajadores Sociales y Asistentes Sociales. Madrid, 1993.

BÁÑEZ TELLO, T., "Situación profesional a la que nos enfrentamos hoy", en DÍ. Aportes desde el Trabajo Social. $N^{\circ} 4 / 5$ (Actas de las Jornadas de Trabajo Social 1995). Colegio Oficial de Diplomados en Trabajo Social y Asistentes Sociales. Alicante, 1995.

BUENO ABAD, J.R., Servicios Sociales: Planificación y organización. Ed. Mestral Llibres. València, 1988.

CAMPANINI, A. y LUPPI, F., Servicio Social y modelo sistémico. Ed. Paidós. Barcelona, 1991.

Curso sobre Mandos Medios. Ayuntamiento de Alicante, 1996. Materiales policopiados.

Consejo General de Colegios Oficiales de Trabajadores Sociales y Asistentes Sociales, "Dictamen sobre definición de objetivos y funciones profesionales", en la Revista de Servicios Sociales y Política Social $n^{\circ} 30$. Consejo General de Colegios Oficiales de Trabajadores Sociales y Asistentes Sociales. Madrid, 1993.

Federación Internacional de Trabajadores Sociales, "La Ética del Trabajo Social: Principios y Criterios". F.I.T.S. Oslo, 1994.

GARCÍA HERRERO, G., Centros de Servicios Sociales. Conceptualización $y$ desarrollo operativo. Ed. Siglo XXI. Madrid, 1988. 
GARCÍA HERRERO, G. Y RAMÍREZ NAVARRO, J.M., Los Nuevos Servicios Sociales. Síntesis de conceptos y evolución. Ed. Certeza. Zaragoza, 1992.

GARCÍA ROCA, J. Público y Privado en la Acción Social. Del Estado de Bienestar al Estado Social. Ed. Popular. Madrid, 1992.

KADUSHIN, A. Supervision in social work. Columbia University Press. New York, 1985.

LÓPEZ CÁBANAS, M. Y GALlEGO GALlEGO, A., "Análisis organizacional y propuestas de desarrollo en los Servicios Sociales Comunitarios", en III Jornadas de Intervención Social. Tomo 1. Ed. INSERSO. Madrid, 1993.

MINTZBERG, Henry, La estructuración de las organizaciones. Ed. Ariel. Barcelona, 1995.

NICHOLS, J. Y CAVE, C., "Supervisión: Algunos principios y consideraciones para la práctica", en Revista de Servicios Sociales y Política Social $n^{\circ} 25$. Consejo General de Colegios Oficiales de Trabajadores Sociales y Asistentes Sociales. Madrid, 1992.

PEIRÓ, J.M ${ }^{\mathrm{a}}$ Y MEDINA, M., "Aspectos psicosociales de la gestión de organizaciones de Servicios Sociales", en IV Jornadas de Intervención Social. Tomo 2. Ed. INSERSO. Madrid, 1996.

PLENCHETTE-BRISSONNET, C., Mètode de Treball en Equip. Ed. Pòrtic/ INTRESS. Barcelona, 1987.

PORCEL MUNDO, A., "La supervisión en Servicios Sociales", en Revista de Servicios Sociales y Política Social $n^{\circ} 25$. Consejo General de Colegios Oficiales de Trabajadores Sociales y Asistentes Sociales. Madrid, 1992.

PORCEL, A. Y VÁZQUEZ, C., La supervisión. Espacio de aprendizaje significativo. Instrumento para la gestión. Ed. Certeza/INTRESS. Zaragoza, 1995.

VILLALBA QUESADA, C. "Redes sociales: Un concepto con importantes implicaciones en la intervención comunitaria", en Intervención Psicosocial. Vol. II. $N^{\circ}$ 4. Madrid, 1993 